\title{
RESULTS FROM 2-D NUMERICAL SIMULATIONS OF SOLAR GRANULES
}

\author{
M. STEFFEN, D. GIGAS, H. HOLWEGER, A. KRÜSS, H.-G. LUDWIG \\ Institut für Theoretische Physik und Sternwarte der Universität Kiel \\ Olshausenstrasse 40 \\ D-2300 Kiel 1 \\ Federal Republic of Germany
}

\section{Calculations}

We have carried out detailed numerical simulations of solar granular convection cells of different horizontal dimension. The calculations account for the basic physics of compressible convection, including the ionization of $\mathrm{HI}, \mathrm{HeI}$ and $\mathrm{He} \mathrm{II}$, and $\mathrm{H}_{2}$ molecule formation as well as non-local, multi-dimensional radiative transfer (grey approximation in LTE). A more detailed description of the simulations has been given by Steffen and Muchmore (1988) and by Steffen et al. (1989).

Recently, the scheme for the computation of the radiation field has been improved to give a better angular resolution and to make sure that numerical integration of the rate of radiative energy exchange, $\operatorname{div} F_{\text {rad }}$, over the model volume gives the same radiative surface flux as obtained directly from integration of the radiative transfer equation along the ray system. This greatly improves the accuracy of the numerical energy conservation, flux errors being of the order of a few per cent, as can be checked easily for steady state models. This is a satisfactory result in view of the steep gradients occurring in the models and the presence of a narrow layer where the energy transport changes from primarily convective to essentially radiative.

\section{Steady State Models}

Steady state solutions are found for model diameters below a critical upper limit. This limit was roughly $2000 \mathrm{~km}$ with the earlier version of the code, while it has not yet been determined with the new radiative transfer scheme, which gives steeper temperature gradients and larger horizontal temperature differences between hot and cool parts of the flow.

We always find a strong downdraft at the axis of symmetry with maximum velocities of the order of $6 \mathrm{~km} / \mathrm{s}$, which is surrounded by a broader ring-like upflow with lower velocity $(\approx 3 \mathrm{~km} / \mathrm{s})$. At the side walls we find another downflow. The convective velocity field extends considerably into the stable atmospheric layers. The rms value of the vertical velocity decreases approximately exponentially with height. The corresponding scale height is of the order of $200 \mathrm{~km}$, the exact value being a function of cell size.

Granulation generates large horizontal temperature differences, reaching typically $4500 \mathrm{~K}$ about $100 \mathrm{~km}$ below the visible surface $(\tau=1)$. Equally remarkable, the calculations produce very steep vertical temperature gradients at the top of the ascending part of the flow (up to $80 \mathrm{~K} / \mathrm{km}$ ) where the hot gas cools rapidly due to efficient radiative energy losses (typically $\left.10^{10}-10^{11} \mathrm{erg} / \mathrm{g} / \mathrm{s}\right)$. The steep temperature gradient in concert with the recombination of 
hydrogen produces a density inversion in this region, i.e. a layer of higher density lies on top of gas with lower density. In the cool intergranular downflows, however, density increases monotonically with depth.

In the overshooting layers the temperature fluctuations change sign. Here the rising gas is cooler than the surrounding sinking parts of the flow. This behavior is a consequence of the penetration of convective motions into stably stratified atmospheric layers. The uppermost layers of the steady state models is essentially in radiative equilibrium; the temperature is almost constant with height as expected for a grey radiative atmosphere. Horizontal temperature fluctuations are insignificant at heights $>300 \mathrm{~km}$ above $\tau=1$. For a detailed study of calculated steady state velocity and temperature fields as a function of horizontal cell size see Steffen et al. (1989).

From detailed radiative transfer calculations we find the rms intensity contrast, $\delta I_{\text {rms }}$, of the 2-dimensional intensity pattern to range typically between 14 and $16 \%$ in the continuum at $5000 \AA$. This value seems in reasonable agreement with observational evidence (e.g. Bray et al., 1984). Towards smaller granular scales the amplitude of the horizontal intensity fluctuations decreases considerably (Steffen et al., 1989). The same is true for the variation of contrast toward the limb. We find a monotonic, roughly linear decrease of $\delta I_{\text {rms }}$ with $\mu$.

As has been demonstrated elsewhere (Steffen, 1987), line bisectors computed from the larger steady state models are in excellent agreement with observations. It must be noted, however, that the convective velocity field of the stationary models is not sufficient to fully explain the observed line broadening.

\section{Non-stationary Models}

Simulations with model diameters exceeding a critical limit never reach a steady state, not even asymptotically. They are truly instationary. Increasing the model diameter beyond this limit, the time evolution of the flow becomes more and more chaotic. It may be considered as a continuous splitting and merging of granules under the constraints imposed by the cylindrical symmetry. Significant changes occur on time scales of the order of 10 minutes (approximately one turnover time), comparable to typical granular lifetimes.

The magnitude of the velocity and temperature fluctuations in the deep photospheric layers is similar to that found in the steady state models. We obtain a time average of the rms intensity contrast that is not significantly different from that found in the steady state models, maximum values at individual instants of time not exceeding $20 \%$. The decrease of contrast towards the limb is, however, significantly less steep than in the steady state models. This is partly due to the somewhat different thermal structure of the photospheric layers. Furthermore, the considerable temporal variations of the emergent mean intensity translate into an additional contribution to the intensity contrast which varies only slightly across the solar disk.

Due to the time dependence of the flow topology the upper photosphere is much more affected by the convective motions than in case of steady state flows. Stochastic up- and downward motions of considerable amplitude are found in the line formation layers. Spatial and temporal averaging of this type of velocity field will probably result in a height dependence of the rms vertical velocity that shows a local minimum somewhere above the $\tau=1$ level with an increase toward higher layers. Furthermore, the non-stationary velocity field should provide sufficient line broadening to explain observations. These points will need further study.

Test calculations have shown that the main effect on the line bisector is to shift it back and forth without seriously distorting its shape; the bisectors more or less keep their C- 
shapes as the flow evolves in time. We never found an inverted C-shape. Therefore, the line bisectors obtained from a time average of the simulations is similar to that derived from comparable steady state models.

It seems evident that the non-stationary models give a more realistic description of the real solar photosphere than steady state flows. In view of the results presented above we have to conclude that the dynamical and thermal structure of the solar photosphere is much different from the situation suggested by flux-constant mixing-length models. In particular, the upper photospheric layers are probably not horizontally homogeneous and their thermal structure is not exclusively determined by radiative equilibrium but also by dynamical phenomena.

\section{Oscillations in Stationary Convection Cells}

As mentioned above, all but the largest model granules studied with the earlier version of the code finally reach a stationary state. Superposed on the convective flow, almost sinusoidal vertical oscillations with a period of about $4 \mathrm{~min}$ are ubiquitous. A preliminary report has been given by Steffen (1988). The oscillation period depends only slightly on model diameter, increasing from about $200 \mathrm{~s}$ to $265 \mathrm{~s}$ over the diameter range $200-2000 \mathrm{~km}$.

In all models the velocity amplitude increases with height, typically from about $\pm 100 \mathrm{~m} / \mathrm{s}$ at the lower boundary to several times that value in photospheric layers. The increase of amplitude with height is less steep than $1 / \sqrt{\rho}$. All layers oscillate in phase. Together, this behavior is compatible with that of evanescent waves. The velocity oscillations are associated with oscillations of temperature that have a non-monotonic height dependence, attaining maximum values of typically $\pm 50 \mathrm{~K}$ in the upper part of the granules where the transition from convective to radiative energy transport occurs.

These oscillations are remarkably persistent. In most models the amplitude remains constant over many periods; some models show a slow decrease, others an increase towards an asymptotic value which never exceeds $500 \mathrm{~m} / \mathrm{s}$. Extensive test calculations have shown that the basic properties of the oscillations are insensitive to details of the numerical scheme such as time step, grid spacing, or SGS viscosity. In addition, the vertical extent of the simulation volume turned out to be not critical.

Which physical properties of the simulated granules determine the frequency, $\omega_{\text {sim }}$, of these oscillations? The striking independence of cell geometry seems to rule out an explanation in terms of a resonant cavity. More likely, $\omega_{\text {sim }}$ is determined by intrinsic properties of the convective flow. Our hypothesis is that $\omega_{\text {sim }}$ is related to the acoustic cut-off frequency, $\omega_{\text {ac }}$, in the upper part of the granule close to $\tau=1$, where both the convective temperature gradient and the temperature oscillations attain their maximum, and the mass density has a local minimum. We suspect that it is here that the driving oscillator may be located. Indeed the value of $\omega_{\text {sim }}(\approx 26 \mathrm{mHz})$ is found to closely coincide with $\omega_{\mathrm{ac}}$ in all model granules. As another test of this hypothesis the dependence of $\omega_{\text {sim }}$ on gravity was investigated. We have run various simulations for solar type stars, keeping $T_{\text {eff }}$ unchanged but varying $\log g$ by up to \pm 0.1 . Indeed $\omega_{\text {sim }}$ turned out to be strictly proportional to gravity. This supports our view that $\omega_{\text {sim }}$ is closely related to $\omega_{\mathrm{ac}}$, which, for an isothermal atmosphere, is given by $\omega_{\mathrm{ac}}=\gamma g / 2 a$.

Our simulations imply that a stationary convective flow is able to generate oscillatory power in the five minute band, most probably through some kind of overstability mechanism. It must be expected that this contributes to the excitation of solar p-modes, in addition to stochastic interaction with instationary convection. Furthermore, an efficient damping mechanism must be active which keeps the amplitude in the linear regime. We 
suspect that radiative transfer at photospheric levels is the cause, but this has still to be studied in detail. It should be noted that the non-stationary simulations show basically the same kind of oscillations, superimposed on the stochastic convective velocity field.

\section{Simulations for other Stars}

Currently attempts are under way to extend our simulations to stars other than the Sun. As a first application the atmosphere of an early A-type main-sequence star of spectral type $\mathrm{A} 0 \mathrm{~V}$ has been choosen. The atmospheric structure of such stars is generally considered to be well understood: convective energy transport is believed to be completely unimportant in the atmospheres of these stars, which are therefore described in terms of static, planeparallel models.

A different picture came to light in the numeric simulations undertaken so far (see also Gigas, 1988, 1989). Although convective energy transport is still unimportant $(\approx 0.5 \%$ of the total flux) in comparison to the energy transported by radiation, mainly vertical gas flows are encountered with velocities considerably larger than the predictions of mixinglength theory. Maximum flow velocities amount to a few hundred meters per second in deep atmospheric layers below the hydrogen ionization zone; similar to the solar case they increase towards smaller, spectroscopically accessible depths up to values of $\approx 2 \mathrm{~km} / \mathrm{s}$. The temporal power spectrum shows a dominant period of $\approx 1070 \mathrm{~s}$, a secondary peak of $\approx 220 \mathrm{~s}$, as well as higher frequency "noise". Like in the solar case, the main period seems to be related to the acoustic cut-off period in the upper part of the computational domain.

Corresponding temperature fluctuations show a maximum of $\approx \pm 500 \mathrm{~K}$ in the region close to the hydrogen ionization zone. Contrary to the velocity field, pressure and temperature fluctuations appear to be in antiphase in the upper and in the lower part of the computational domain with a common phase shift of $\approx \pi / 2$ relative to the velocity field.

The presence of such oscillatory velocity fields in the atmospheres of early-type stars may provide a satisfactory explanation for the microturbulence parameter $\xi$, which has to be employed in abundance analyses of such stars even if deviations from LTE are taken into account. Typically, microturbulence values of $\approx 1.0-2.0 \mathrm{~km} / \mathrm{s}$ are required, which is in satisfactory agreement with the results encountered in our simulations so far. We are planning to extend these computations to stars of other effective temperature and gravity.

\section{References}

Bray, R.J., Loughhead, R.E., Durrant, C.J.: 1984, in: The Solar Granulation, Cambridge University Press, 2nd ed.

Gigas, D.: 1988, in: The Impact of Very High S/N Spectroscopy on Stellar Physics, Proc. IAU Symposium No. 132, eds. G. Cayrel de Strobel, M. Spite, p. 395

Gigas, D.: 1989, in: Solar and Stellar Granulation, eds. R.J. Rutten, G. Severino, Kluwer, p. 533

Steffen, M.: 1987, in: The Role of Fine-Scale Magnetic Fields on the Structure of the Solar Atmosphere, eds. E.-H. Schröter, M. Vazquez, A.A. Wyller, Cambridge University Press, p. 47

Steffen, M.: 1988, in: Advances in Helio- and Asteroseismology, Proc. IAU Symposium No. 123, eds. J. Christensen-Dalsgaard, S. Frandsen, p. 379

Steffen, M., Muchmore, D.: 1988, Astron. Astrophys. 193, 281

Steffen, M., Ludwig, H.-G., Krüß, A.: 1989, Astron. Astrophys. 213, 371 